\title{
INVERSE THEOREMS FOR SUBSET SUMS
}

\author{
MELVYN. B. NATHANSON
}

\begin{abstract}
Let $A$ be a finite set of integers. For $h \geq 1$, let $S_{h}(A)$ denote the set of all sums of $h$ distinct elements of $A$. Let $S(A)$ denote the set of all nonempty sums of distinct elements of $A$. The direct problem for subset sums is to find lower bounds for $\left|S_{h}(A)\right|$ and $|S(A)|$ in terms of $|A|$. The inverse problem for subset sums is to determine the structure of the extremal sets $A$ of integers for which $\left|S_{h}(A)\right|$ and $|S(A)|$ are minimal. In this paper both the direct and the inverse problem for subset sums are solved.
\end{abstract}

\section{INTRODUCTION}

Let $A=\left\{a_{0}, a_{1}, \ldots, a_{k-1}\right\}$ be a finite set of integers, and let $|A|=k$. For any nonempty subset $A^{\prime}$ of $A$ we define the subset sum $s\left(A^{\prime}\right)$ by

$$
s\left(A^{\prime}\right)=\sum_{a \in A^{\prime}} a .
$$

Let $s(\varnothing)=0$. For $h=0,1,2, \ldots, k$ let

$$
S_{h}(A)=\left\{s\left(A^{\prime}\right)\left|A^{\prime} \subseteq A,\right| A \mid=h\right\} .
$$

The set $S_{h}(A)$ is the set of all sums of $h$ distinct elements of $A$. In particular, $S_{0}(A)=\{0\}, S_{1}(A)=A$, and $S_{k}(A)=\left\{a_{0}+a_{1}+\cdots+a_{k-1}\right\}$. The set

$$
S(A)=\bigcup_{h=1}^{k} S_{h}(A)=\left\{s\left(A^{\prime}\right) \mid \varnothing \neq A^{\prime} \subseteq A\right\}
$$

is the set of all nonempty sums of distinct elements of $A$. The direct problem for subset sums is to find lower bounds for $\left|S_{h}(A)\right|$ and $|S(A)|$ in terms of $|A|$. The inverse problem for subset sums is determine the structure of the finite sets $A$ of integers for which $\left|S_{h}(A)\right|$ and $|S(A)|$ are minimal. In this paper we shall solve both the direct and the inverse problem for subset sums.

Notation. For any finite set $A$ of integers, let $|A|$ denote the cardinality of $A$, let $\max (A)$ denote the largest element of $A$, and let $\min (A)$ denote the

Received by the editors January 30,1994; originally communicated to the Proceedings of the $A M S$ by William Adams.

1991 Mathematics Subject Classification. Primary 11B13, 11B75, 11 P99.

Key words and phrases. Additive number theory, subset sums, inverse theorems.

This work was supported in part by grants from the PSC-CUNY Research Award Program. The paper was written while I was a visitor at the Center for Discrete Mathematics and Theoretical Computer Science (DIMACS) at Rutgers University, and I thank DIMACS for its hospitality. 
smallest element of $A$. If $A^{\prime} \subseteq A$, then $A \backslash A^{\prime}$ denotes the complement of $A^{\prime}$ in $A$. For any integer $d$, let

$$
d+A=\{d+a \mid a \in A\} \text { and } d * A=\{d a \mid a \in A\} .
$$

Let $[u, v]$ denote the interval of integers $n$ such that $u \leq n \leq v$. A $k$-term arithmetic progression is a set of the form

$$
\left\{a_{0}, a_{0}+d, a_{0}+2 d, \ldots, a_{0}+(k-1) d\right\}=a_{0}+d *[0, k-1],
$$

where $a_{0}$ and $d$ are integers and $d \geq 1$. The integer part of the real number $x$ is denoted $[x]$.

\section{THE DIRECT PROBLEM FOR $S_{h}(A)$}

Let $h \in[0, k]$ and let $A^{\prime} \subseteq A,\left|A^{\prime}\right|=h$. Then $\left|A \backslash A^{\prime}\right|=k-h$ and

$$
s(A)-s\left(A^{\prime}\right)=s\left(A \backslash A^{\prime}\right) .
$$

This identity establishes a natural bijection

$$
\chi: S_{h}(A) \rightarrow S_{k-h}(A)
$$

defined by

$$
\chi\left(s\left(A^{\prime}\right)\right)=s\left(A \backslash A^{\prime}\right) .
$$

It follows that

$$
\left|S_{h}(A)\right|=\left|S_{k-h}(A)\right|
$$

for $h=0,1, \ldots, k$.

Theorem 1. Let $A$ be a set of $k$ integers, and let $h \in[0, k]$. Then

$$
\left|S_{h}(A)\right| \geq h k-h^{2}+1=h(k-h)+1 .
$$

Proof. Let $A=\left\{a_{0}, a_{1}, \ldots, a_{k-1}\right\}$, where $a_{0}<a_{1}<\cdots<a_{k-1}$. For $i=$ $0,1, \ldots, k-h-1$ and $j=0,1, \ldots, h$, we define

$$
s_{i, j}^{(h)}=s_{i, j}=\sum_{\substack{l=0 \\ l \neq h-j}}^{h} a_{i+l}
$$

and

$$
s_{k-h, 0}^{(h)}=s_{k-h, 0}=\sum_{l=0}^{h-1} a_{k-h+l} .
$$

Each of these numbers is a sum of $h$ distinct elements of $A$, and so $s_{i, j} \in S_{h}(A)$ for all $i$ and $j$. Moreover, for $i=0,1, \ldots, k-h-1$ and $j=0,1, \ldots, h-1$, we have

$$
s_{i, j+1}-s_{i, j}=a_{i+h-j}-a_{i+h-j-1}>0
$$

and

$$
s_{i, h}=\sum_{l=1}^{h} a_{i+l}=\sum_{l=0}^{h-1} a_{i+1+l}=s_{i+1,0} .
$$

Therefore,

$$
s_{i, 0}<s_{i, 1}<s_{i, 2}<\cdots<s_{i, h-1}<s_{i, h}=s_{i+1,0}
$$


and so

$$
\left|S_{h}(A)\right| \geq h(k-h)+1=h k-k^{2}+1 .
$$

This completes the proof.

\section{The INVERSE PROBLEM FOR $S_{h}(A)$}

If $A=[0, k-1]$, then

$$
S_{h}(A)=\left[\left(\begin{array}{l}
h \\
2
\end{array}\right), h k-\left(\begin{array}{c}
h+1 \\
2
\end{array}\right)\right]=\left(\begin{array}{l}
h \\
2
\end{array}\right)+\left[0, h k-h^{2}\right]
$$

and so

$$
\left|S_{h}(A)\right|=h k-h^{2}+1=h(k-h)+1 .
$$

This shows that the lower bound in Theorem 1 is best possible. The inverse problem for $S_{h}(A)$ is to find all sets $A$ of $k$ integers that satisfy (6).

If $a$ and $d$ are integers and $d \neq 0$, then

$$
S_{h}(a+d * A)=h a+d * S_{h}(A),
$$

and so

$$
\left|S_{h}(a+d * A)\right|=\left|S_{h}(A)\right| .
$$

This means that the function $\left|S_{h}(A)\right|$ is an affine invariant of the set $A$. Since every interval of length $k$ satisfies condition (6), it follows from (1) and (7) that every $k$-term arithmetic progression also satisfies (6).

Let $|A|=k$ and $h \in[0, k]$. The symmetry (3) implies that if $A$ is an arithmetic progression whenever $\left|S_{h}(A)\right|$ satisfies (6), then $A$ is also an arithmetic progression whenever $\left|S_{k-h}(A)\right|$ satisfies (6).

Not all extremal sets are arithmetic progressions. Here are some examples:

(i) If $h=0$ or $h=k$, then $h(k-h)+1=1$ and $\left|S_{0}(A)\right|=S_{h}(A) \mid=1$ for every set $A$.

(ii) If $h=1$ or $h=k-1$, then $h(k-h)+1=k$ and $\left|S_{1}(A)\right|=\left|S_{h-1}(A)\right|=$ $k$ for every set $A$.

(iii) If $h=2$ and $k=4$, then $h(k-h)+1=5$. Let

$$
A=\left\{a_{0}, a_{1}, a_{2}, a_{3}\right\}
$$

be a set of integers such that $a_{0}<a_{1}<a_{2}<a_{3}$. Then

$$
S_{2}(A)=\left\{a_{0}+a_{1}, a_{0}+a_{2}, a_{0}+a_{3}, a_{1}+a_{2}, a_{1}+a_{3}, a_{2}+a_{3}\right\}
$$

and so $5 \leq\left|S_{2}(A)\right| \leq 6$. Since

$$
a_{0}+a_{1}<a_{0}+a_{2}<a_{0}+a_{3}<a_{1}+a_{3}<a_{2}+a_{3}
$$

and

$$
a_{0}+a_{2}<a_{1}+a_{2}<a_{1}+a_{3}
$$

it follows that $\left|S_{2}(A)\right|=5$ if and only if

$$
a_{0}+a_{3}=a_{1}+a_{2} \text {. }
$$

Thus, $\left\{a_{0}, a_{1}, a_{2}, a_{2}+a_{1}-a_{0}\right\}$ is an extremal set for all $a_{0}<a_{1}<a_{2}$. We shall prove that these three examples are the only examples of extremal sets for $\left|S_{h}(A)\right|$ that are not arithmetic progressions. 
Theorem 2. Let $k \geq 5$ and let $2 \leq h \leq k-2$. If $A$ is a set of $k$ integers such that

$$
\left|S_{h}(A)\right|=h k-h^{2}+1,
$$

then $A$ is an arithmetic progression.

Proof. Let $A=\left\{a_{0}, a_{1}, \ldots, a_{k-1}\right\}$, where

$$
a_{0}<a_{1}<\cdots<a_{k-1} \text {. }
$$

It follows from the proof of Theorem 1 that the set $A$ consists of the numbers $s_{i, j}$ defined in (4) and (5). Let $i=0,1, \ldots, k-h-2$ and $j=2,3, \ldots, h$. Then

$$
s_{i, j}=\sum_{\substack{l=0 \\ l \neq h-j}}^{h-1} a_{i+l}+a_{i+h}
$$

and

$$
s_{i, 1}<s_{i, 2}<s_{i, 3}<\cdots<s_{i, h}=s_{i+1,0}<s_{i+1,1} .
$$

Consider the integers

$$
u_{i, j}=\sum_{\substack{l=0 \\ l \neq h+1-j}}^{h-1} a_{i+l}+a_{i+h+1} \in S_{h}(A) .
$$

Since

$$
s_{i, 1}<u_{i, 2}<u_{i, 3}<\cdots<u_{i, h}<s_{i+1,1},
$$

it follows that $s_{i, j}=u_{i, j}$ and so

$$
a_{i+h-j+1}+a_{i+h}=a_{i+h-j}+a_{i+h+1}
$$

for $i=0,1 \ldots, k-h-2$ and $j=2,3, \ldots, h$. Thus,

$$
a_{i+h-j+1}-a_{i+h-j}=a_{i+h+1}-a_{i+h}
$$

and

$$
\begin{aligned}
a_{i+1}-a_{i} & =a_{i+2}-a_{i+1}=\cdots \\
& =a_{i+h-2}-a_{i+h-3} \\
& =a_{i+h-1}-a_{i+h-2} \\
& =a_{i+h+1}-a_{i+h}
\end{aligned}
$$

for $i=0,1, \ldots, k-h-2$. We shall show that

$$
a_{i+h}-a_{i+h-1}=a_{i+1}-a_{i} \text {. }
$$

For any $i \geq 1$ we have

$$
\begin{aligned}
a_{i+h}-a_{i+h-1} & =a_{i-1+(h+1)}-a_{i-1+h} \\
& =a_{i-1+(h-1)}-a_{i-1+(h-2)} \\
& =a_{i+h-2}-a_{i+h-3} \\
& =a_{i+1}-a_{i} .
\end{aligned}
$$


Let $i=0$. We must show that $a_{h}-a_{h-1}=a_{1}-a_{0}$. If $h<k-2$, then $1 \leq k-h-2$ and

It follows that

$$
\begin{aligned}
a_{h}-a_{h-1} & =a_{1+(h-1)}-a_{1+(h-2)} \\
& =a_{1+(h-2)}-a_{1+(h-3)} \\
& =a_{h-1}-a_{h-2} \\
& =a_{1}-a_{0} .
\end{aligned}
$$

$$
a_{i}-a_{i-1}=a_{1}-a_{0}
$$

for $i=0,1, \ldots, k-1$, and so $A$ is an arithmetic progression. If $h=k-2$, then we consider $\left|S_{2}(A)\right|$. By (3),

$$
\left|S_{2}(A)\right|=\left|S_{k-2}(A)\right|=2(k-2)+1 .
$$

Since $2<k-2$ for $k \geq 5$, it follows that $A$ is an arithmetic progression. This completes the proof.

\section{THE DIRECT PROBLEM FOR $S(A)$}

Theorem 3. Let $k \geq 2$. If $A$ is a set of $k$ positive integers, then

$$
|S(A)| \geq\left(\begin{array}{c}
k+1 \\
2
\end{array}\right)
$$

If $A$ is a set of $k$ nonnegative integers and $0 \in A$, then

$$
|S(A)| \geq 1+\left(\begin{array}{l}
k \\
2
\end{array}\right)
$$

Proof. Let $A=\left\{a_{0}, a_{1}, \ldots, a_{k-1}\right\}$, where $a_{0}<a_{1}<\cdots<a_{k-1}$. For $h=$ $1, \ldots, k$, let

$$
B_{h}=\left\{a_{i}+a_{k-h+1}+a_{k-h+2}+\cdots+a_{k-1} \mid i=0,1 \ldots, k-h\right\} .
$$

Then

and

$$
B_{h} \subseteq S_{h}(A) \subseteq S(A)
$$

$$
\left|B_{h}\right|=k-h+1 \text {. }
$$

If $A$ is a set of positive integers, then $a_{0} \geq 1$ and

$$
\begin{aligned}
\max \left(B_{h}\right) & =a_{k-h}+a_{k-h+1}+\cdots+a_{k-1} \\
& <a_{0}+a_{k-h}+a_{k-h+1}+\cdots+a_{k-1} \\
& =\min \left(B_{h+1}\right) .
\end{aligned}
$$

It follows that the sets $B_{1}, B_{2}, \ldots, B_{k}$ are pairwise disjoint, and so

$$
|S(A)| \geq\left|\bigcup_{h=1}^{k} B_{h}\right|=\sum_{h=1}^{k}\left|B_{h}\right|=\sum_{h=1}^{k}(k-h+1)=\left(\begin{array}{c}
k+1 \\
2
\end{array}\right) .
$$

If $A$ is a set of nonnegative integers and $a_{0}=0$, then

$$
S(A)=\{0\} \cup S(A \backslash\{0\})
$$

and $A \backslash\{0\}$ is a set of $k-1$ positive integers. Therefore,

$$
|S(A)| \geq 1+|S(A \backslash\{0\})| \geq 1+\left(\begin{array}{l}
k \\
2
\end{array}\right) .
$$

This completes the proof. 
Let $k \geq 2$. If $A_{0}=[0, k-1]$ and $A_{1}=[1, k]$, then

$$
S\left(A_{0}\right)=\left[0,\left(\begin{array}{l}
k \\
2
\end{array}\right)\right] \text { and } S\left(A_{1}\right)=\left[1,\left(\begin{array}{c}
k+1 \\
2
\end{array}\right)\right] \text {. }
$$

These examples show that the lower bounds in Theorem 3 are best possible.

Theorem 4. Let $k \geq 2$, and let $A$ be a set of $k$ integers. If $0 \in A$, then

$$
|S(A)| \geq\left[k^{2} / 4\right]+1 \text {. }
$$

If $0 \notin A$, then

$$
|S(A)| \geq\left[(k+1)^{2} / 4\right]+1 .
$$

Proof. If $|A|=2$, then $|S(A)|=2$ if $0 \in A$ and $|S(A)|=3$ if $0 \notin A$. Let $k \geq 3$. Since $\left(\begin{array}{l}k \\ 2\end{array}\right)>k^{2} / 4$ for $k \geq 3$, we can assume that $A$ contains both positive and negative integers. Let $A$ contain $p$ positive integers and $n$ negative integers. By Theorem 3 , the set $S(A)$ contains at least $\left(\begin{array}{c}p+1 \\ 2\end{array}\right)$ positive integers and at least $\left(\begin{array}{c}n+1 \\ 2\end{array}\right)$ negative integers. If $0 \in A \subseteq S(A)$, then $k=p+n+1$ and

$$
\begin{aligned}
|S(A)| & \geq\left(\begin{array}{c}
p+1 \\
2
\end{array}\right)+\left(\begin{array}{c}
n+1 \\
2
\end{array}\right)+1 \\
& =\left(\begin{array}{c}
p+1 \\
2
\end{array}\right)+\left(\begin{array}{c}
k-p \\
2
\end{array}\right)+1 \\
& =p^{2}-(k-1) p+\left(k^{2}-k+2\right) / 2 \\
& =(p-(k-1) / 2)^{2}+\left(k^{2}+3\right) / 4 \\
& \geq\left(k^{2}+3\right) / 4,
\end{aligned}
$$

and so $|S(A)| \geq\left[k^{2} / 4\right]+1$.

If $0 \notin A$, then

$$
k=p+n .
$$

Let $p_{0}$ be the smallest positive integer in $A$, and let $-n_{0}$ be the largest negative integer in $A$. The set $S(A)$ contains at least $\left(\begin{array}{c}p+1 \\ 2\end{array}\right)$ positive numbers, each greater than or equal to $p_{0}$, and $S(A)$ contains at least $\left(\begin{array}{c}n+1 \\ 2\end{array}\right)$ negative numbers, each less than or equal to $-n_{0}$. The set $S(A)$ also contains the integer $p_{0}-n_{0}$. Since

$$
-n_{0}<p_{0}-n_{0}<p_{0},
$$

it follows that

$$
\begin{aligned}
|S(A)| & \geq\left(\begin{array}{c}
p+1 \\
2
\end{array}\right)+\left(\begin{array}{c}
n+1 \\
2
\end{array}\right)+1 \\
& =\left(\begin{array}{c}
p+1 \\
2
\end{array}\right)+\left(\begin{array}{c}
k-p+1 \\
2
\end{array}\right)+1 \\
& =p^{2}-k p+\left(k^{2}+k+2\right) / 2 \\
& =(p-k / 2)^{2}+\left((k+1)^{2}+3\right) / 4 \\
& \geq\left((k+1)^{2}+3\right) / 4,
\end{aligned}
$$

and so

$$
|S(A)| \geq\left[(k+1)^{2} / 4\right]+1 .
$$

This completes the proof. 
Let $k=2 m$ be an even integer. If $A=[-m, m-1]$, then $|A|=k, 0 \in A$, and

$$
S(A)=\left[-\left(\begin{array}{c}
m+1 \\
2
\end{array}\right),\left(\begin{array}{c}
m \\
2
\end{array}\right)\right]
$$

hence

$$
|S(A)|=\left(\begin{array}{c}
m+1 \\
2
\end{array}\right)+\left(\begin{array}{c}
m \\
2
\end{array}\right)+1=m^{2}+1=\left[k^{2} / 4\right]+1 .
$$

If $A=[-m, m] \backslash\{0\}$, then $|A|=k, 0 \notin A$, and

$$
S(A)=\left[-\left(\begin{array}{c}
m+1 \\
2
\end{array}\right),\left(\begin{array}{c}
m+1 \\
2
\end{array}\right)\right]
$$

hence

$$
|S(A)|=2\left(\begin{array}{c}
m+1 \\
2
\end{array}\right)+1=m^{2}+m+1=\left[(k+1)^{2} / 4\right]+1 .
$$

Let $k=2 m+1$ be an odd integer. If $A=[-m, m]$, then $|A|=k, 0 \in A$, and

$$
S(A)=\left[-\left(\begin{array}{c}
m+1 \\
2
\end{array}\right),\left(\begin{array}{c}
m+1 \\
2
\end{array}\right)\right]
$$

hence

$$
|S(A)|=2\left(\begin{array}{c}
m \\
2
\end{array}\right)+1=m^{2}+m+1=\left(k^{2}+3\right) / 4=\left[k^{2} / 4\right]+1 .
$$

Finally, if $A=[-m, m+1] \backslash\{0\}$, then $|A|=k, 0 \notin A$, and

$$
S(A)=\left[-\left(\begin{array}{c}
m+1 \\
2
\end{array}\right),\left(\begin{array}{c}
m+2 \\
2
\end{array}\right)\right]
$$

hence

$$
|S(A)|=\left(\begin{array}{c}
m+1 \\
2
\end{array}\right)+\left(\begin{array}{c}
m+2 \\
2
\end{array}\right)+1=m^{2}+2 m+2=\left[(k+1)^{2} / 4\right]+1 .
$$

These examples show that the lower bounds in Theorem 4 are best possible.

5. THE INVERSE PROBLEM FOR $S(A)$

Let $k \geq 3$ and let $A_{0}=[0, k-1]$ and $A_{1}=1+A_{0}=[1, k]$. Since

$$
\left|S\left(A_{0}\right)\right|=1+\left(\begin{array}{c}
k \\
2
\end{array}\right)<\left(\begin{array}{c}
k+1 \\
2
\end{array}\right)=\left|S_{1}(A)\right|
$$

we see that $|S(A)|$ is not translation invariant. However, if $d$ is a nonzero integer, then

$$
S(d * a)=d * S(A)
$$

and so

$$
|S(d * A)|=|S(A)| .
$$

Thus, $|S(A)|$ is invariant under scalar multiplication.

The inverse problem for $S(A)$ is to find the extremal sets $A$ that satisfy the lower bounds in Theorem 3 and in Theorem 4 . We shall prove that every extremal set is a scalar multiple of an interval. 
Theorem 5. Let $k \geq 3$. If $A$ is a set of $k$ positive integers such that $|S(A)|=$ $\left(\begin{array}{c}k+1 \\ 2\end{array}\right)$, then

$$
A=d *[1, k]
$$

for some positive integer $d$. If $A$ is a set of $k$ nonnegative integers such that $0 \in A$ and $|S(A)|=1+\left(\begin{array}{l}k \\ 2\end{array}\right)$, then

$$
A=d *[0, k-1]
$$

for some positive integer $d$.

Proof. Let $A=\left\{a_{0}, a_{1}, \ldots, a_{k-1}\right\}$, where $a_{0}<a_{1}<\cdots<a_{k-1}$. If $a_{0} \geq 1$, then it follows from the proof of Theorem 3 that

$$
S(A)=\bigcup_{i=1}^{h} B_{h},
$$

where $B_{h}$ is the set defined in (8). For $h=1, \ldots, k-1$, we have

$$
\begin{aligned}
a_{k-h-1} & +a_{k-h+1}+a_{k-h+2}+\cdots+a_{k-1} \\
& <a_{k-h}+a_{k-h+1}+a_{k-h+2}+\cdots+a_{k-1}=\max \left(B_{h}\right) \\
& <a_{0}+a_{k-h}+a_{k-h+1}+a_{k-h+2}+\cdots+a_{k-1}=\min \left(B_{h+1}\right)
\end{aligned}
$$

and

$$
\begin{aligned}
a_{k-h-1} & +a_{k-h+1}+a_{k-h+2}+\cdots+a_{k-1} \\
& <a_{0}+a_{k-h-1}+a_{k-h+1}+a_{k-h+2}+\cdots+a_{k-1} \\
& <a_{0}+a_{k-h}+a_{k-h+1}+a_{k-h+2}+\cdots+a_{k-1} .
\end{aligned}
$$

It follows that

$$
\begin{aligned}
a_{k-h} & +a_{k-h+1}+a_{k-h+2}+\cdots+a_{k-1} \\
& =a_{0}+a_{k-h-1}+a_{k-h+1}+a_{k-h+2}+\cdots+a_{k-1}
\end{aligned}
$$

and so

$$
a_{k-h}=a_{0}+a_{k-h-1}
$$

for $h=1, \ldots, k-1$. Thus,

$$
a_{0}=a_{1}-a_{0}=a_{2}-a_{1}=\cdots=a_{k-1}-a_{k-2}
$$

and so

$$
A=d *[1, k]
$$

with $d=a_{0}$.

If $a_{0}=0$, then $A \backslash\{0\}$ is a set of $k-1$ positive integers, and $S(A \backslash\{0\})$ is a set of positive integers. Since

$$
S(A)=\{0\} \cup S(A \backslash\{0\}),
$$

it follows that

$$
|S(A \backslash\{0\})|=\left(\begin{array}{l}
k \\
2
\end{array}\right)
$$

and so $A \backslash\{0\}=d *[1, k-1]$ for $d=a_{1} \geq 1$. Thus,

$$
A=d *[0, k-1] \text {. }
$$

This completes the proof. 
Theorem 6. Let $k \geq 3$, and let $A$ be a set of $k$ integers. If $0 \in A$ and $|S(A)|=\left[k^{2} / 4\right]+1$, then there is a nonzero integer $d$ such that

$$
A= \begin{cases}d *[-m, m] & \text { if } k=2 m+1, \\ d *[-m, m-1] & \text { if } k=2 m .\end{cases}
$$

If $0 \notin A$ and $|S(A)|=\left[(k+1)^{2} / 4\right]+1$, then there is a nonzero integer $d$ such that

$$
A= \begin{cases}d *[-m, m] \backslash\{0\} & \text { if } k=2 m, \\ d *[-m, m+1] \backslash\{0\} & \text { if } k=2 m+1 .\end{cases}
$$

Proof. The conditions on $|S(A)|$ imply that $A$ contains $p$ positive and $n$ negative integers, where $p \geq 1$ and $n \geq 1$. If $0 \in A$, then $k=p+n+1$ and

$$
\begin{aligned}
{\left[k^{2} / 4\right]+1 } & =|S(A)| \\
& \geq\left(\begin{array}{c}
p+1 \\
2
\end{array}\right)+\left(\begin{array}{c}
n+1 \\
2
\end{array}\right)+1 \\
& =\left(\begin{array}{c}
p+1 \\
2
\end{array}\right)+\left(\begin{array}{c}
k-p \\
2
\end{array}\right)+1 \\
& =(p-(k-1) / 2)^{2}+\left(k^{2}+3\right) / 4 \\
& \geq\left(k^{2}+3\right) / 4 .
\end{aligned}
$$

It follows that

$$
|S(A)|=1+\left(\begin{array}{c}
p+1 \\
2
\end{array}\right)+\left(\begin{array}{c}
n+1 \\
2
\end{array}\right)
$$

and

that is,

$$
p-(k-1) / 2= \begin{cases}0 & \text { if } k=2 m+1 \text { is odd } \\ \pm 1 / 2 & \text { if } k=2 m \text { is even }\end{cases}
$$

$$
p= \begin{cases}m & \text { if } k=2 m+1 \text { is odd } \\ m \text { or } m-1 & \text { if } k=2 m \text { is even. }\end{cases}
$$

Suppose that $k=2 m+1$. Then $p=n=m$. Since $|S(A)|$ contains exactly $\left(\begin{array}{c}m+1 \\ 2\end{array}\right)$ positive integers and exactly $\left(\begin{array}{c}m+1 \\ 2\end{array}\right)$ negative integers, it follows from Theorem 5 that there exist positive integers $p_{0}$ and $n_{0}$ such that the positive part of $A$ is $p_{0} *[1, m]$ and the negative part of $A$ is $-n_{0} *[1, m]$. Since $0, p_{0}-n_{0} \in S(A)$ and $-n_{0}<p_{0}-n_{0}<p_{0}$, it follows that $p_{0}-n_{0}=0$. Let $d=p_{0}$. Then

$$
A=d *[-m, m]
$$

The proof in the case $k=2 m$ is similar.

If $0 \notin A$, then $k=p+n$ and

$$
\begin{aligned}
{\left[(k+1)^{2} / 4\right]+1 } & =|S(A)| \geq\left(\begin{array}{c}
p+1 \\
2
\end{array}\right)+\left(\begin{array}{c}
n+1 \\
2
\end{array}\right)+1 \\
& =\left(\begin{array}{c}
p+1 \\
2
\end{array}\right)+\left(\begin{array}{c}
k-p+1 \\
2
\end{array}\right)+1 \\
& =(p-(k / 2))^{2}+\left((k+1)^{2}+3\right) / 4 \\
& \geq\left((k+1)^{2}+3\right) / 4,
\end{aligned}
$$

and the proof proceeds as above. 


\section{REMARKS}

There is a large and growing literature on subset sums (for example, Sárközy [4]) and on inverse problems in additive number theory, but the inverse theorems on subset sums do not seem to have been previously observed. It would be interesting to extend these results in the following way: Let $A$ be a set of $k$ integers such that $\left|S_{h}(A)\right|$ is "small." Is $A$ a "large" subset of some arithmetic progression? If $|A|=k$ and if $2 A$ denotes the set of all sums of 2 elements of $A$ with repetitions allowed, and if $|2 A|=2 k-1+b \leq 3 k-4$, then Freiman [1] (see also Nathanson [2]) proved that $A$ is a subset of an arithmetic progression of length $k+b$. Does a similar result hold for subset sums?

A comprehensive account of inverse theorems in additive number theory can be found in Nathanson [3].

\section{REFERENCES}

1. G. A. Freiman, On the addition of finite sets. I, Izv. Vyssh. Uchebn. Zaved. Mat. 13 (1959), 202-213.

2. M. B. Nathanson, The simplest inverse problems in additive number theory, Number Theory with an Emphasis on the Markoff Spectrum (A. Pollington and W. Moran, eds.), Marcel Dekker, 1993, pp. 191-206.

3. _ Additive number theory: 2 Inverse theorems and the geometry of sumsets, SpringerVerlag, New York, 1995.

4. A. Sárközy, Finite addition theorems. II, J. Number Theory 48 (1994), 197-218.

Department of Mathematics, Lehman College (CUNY), Bronx, New York 10468

E-mail address: nathansn@dimacs.rutgers .edu 